\title{
Canadian Journal of Plastic Surgery's momentum continues in 2013
}

$\mathrm{O}^{\mathrm{n}}$ ne year ago, the Canadian Journal of Plastic Surgery (CJPS) announced the beginning of a new and exciting phase in the Journal's history. In collaboration with Pulsus Group Inc, and the continued support of the Canadian Society of Plastic Surgeons, our sponsors, advertisers and readers, we have made significant advancements over the past year. Issues in excess of 60 pages, the introduction of new sections, including educational material, $\mathrm{CME}$, financial and practice management, invited special topics and the implementation of level of evidence rating to the online submission system are a few of the Journal's accomplishments in the past year. The goal of the Journal is to continue this momentum into the years to come.

One of the ways the Journal plans to achieve this goal is to increase accessibility and readership. In 2013, the primary modality for this will be the introduction of the CJPS App.

CJPS is proud to announce the release of its own App. The App is FREE and available at the Apple App Store now (https://itunes.apple. com/us/app/canadian-journal-plastic-surgery/id592183537?mt=8). The App provides quick access to the complete CJPS (some articles may require subscriber login).

The App allows you to:

- Browse, filter, and search multiple volumes and their issues.

- Download full-text articles for offline reading

- Automatically receive new issues as they become available.

- View surgical demonstration videos from full-text articles.

- Bookmark articles and make notes on articles. E-mail yourself a list of all your notes.

- Review bookmarks and notes or recently accessed articles in 'Viewed' tab.

- Follow the \#CJPS Twitter feed and post your own opinion about an article on Twitter.

- Share article information on social media.

In addition to the Apple App, the Journal will be releasing an 'Android' version of the App shortly. Thank you to our sponsors, advertisers, members and the Canadian Society of Plastic Surgeons for their continued support as we develop the CJPS.

Sincerely, Edward W Buchel MD 